\title{
From Nuggets of SQM to Domains of Broken Symmetry (In memoriam of Jack Sandweiss)
}

\author{
Evan Finch $^{1, *}$ \\ ${ }^{1}$ Southern Connecticut State University
}

\begin{abstract}
For the last 20 years of his remarkable career, Jack Sandweiss was an enthusiastic leader in heavy-ion physics, especially excited about two main topics: the search for stable or metastable strange quark matter and the possibility of local parity violation in heavy-ion collisions. I take a look back at some of Jack's contributions in the context of the current state of both of these very active areas.
\end{abstract}

\section{Jack's early career}

It was an honor for me to be able to give this talk remembering Jack Sandweiss at the SQM '21 conference. I had the great pleasure of working in Jack's group at Yale for about twenty years and have extremely fond memories of working with him, Dick Majka and Alexei Chikanian among the many great personalities of the 5th floor of Gibbs Lab. Anyone who worked with Jack remembers his truly extraordinary broad and deep knowledge of physics, but what stands out to many of us even more was his relentless enthusiasm, not just for his field or for physics in general, but for just about everything he was involved with, and just how much he enjoyed talking to other people about what they were excited about to broaden his own interests. John Harris, who was chairing this session at SQM, shared how Jack's presence was one of the major draws for John coming to Yale, and also remembered Jack's as a "true academic", a term that really fit Jack with his vast interests and the involvements he shared with his wife Letha as it does few others.

Jack's graduate studies were done at the rad lab in Berkeley, and he started out right in the thick of particle physics, where he worked in one of the groups searching for the antiproton, and though their technique didn't win the race due to the unexpectedly large annihilation cross-section, they were able to make one of the first measurements of that cross section [1]. He did a bit of work [2] that contributed to the understanding of the " $\tau-\theta$ " kaon puzzle that helped lead to the discovery of weak parity violation. He did it all - he soon moved to Yale where he worked with one of his first students Charlie Baltay as they became experts on building RF-field separated beams at Brookhaven to isolate beams of kaons and hyperons. And one of the accomplishments he often spoke of most fondly was the design and construction of the high-resolution streamer chamber for charm quark measurements at Fermilab [3]. He became an eminently respected physicist; helping to steer the field by chairing the HEPAP advisory panel in the 80s, and succeeding his friend Bob Adair as chief editor of Physical Review Letters through the 90s and 2000s.

\footnotetext{
*e-mail: FinchL3@ southernCT.edu
} 


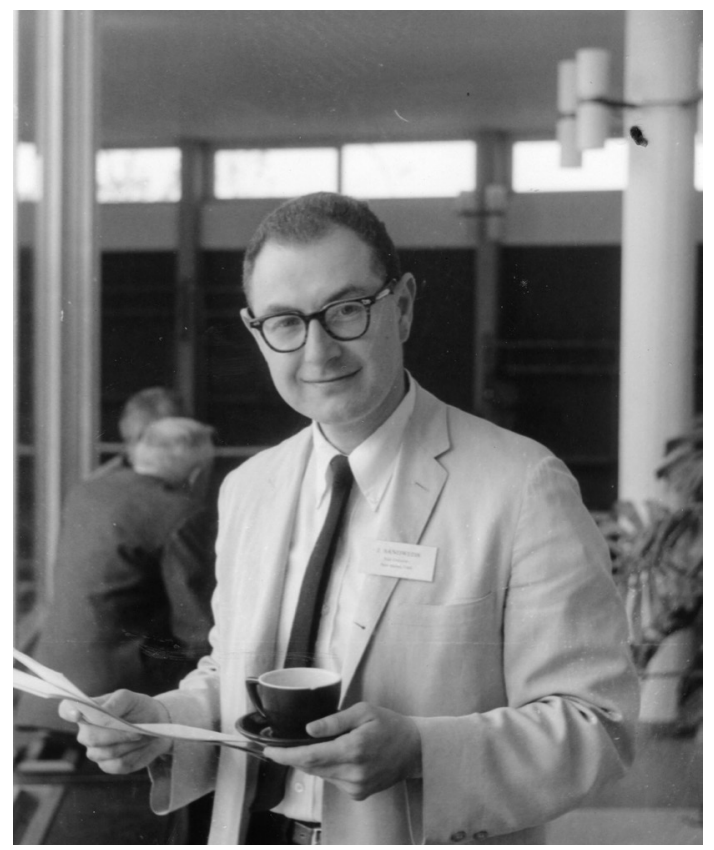

Figure 1. Jack at CERN. AIP Emilio Segre Visual Archives, Segre Collection

\section{Searches for SQM}

Jack's interest in searches for stable Strange Quark Matter (SQM) began in the mid-80s. SQM, made up of roughly $1 / 3$ up, down, and strange quarks in a single hadronic state with baryon number $A \geq 2$, was suggested almost 50 years ago as the possible true ground state of nuclear matter $[4,5]$. That quark matter with only up and down quarks could be the true ground state of hadronic matter at zero temperature and pressure is easily dismissed by the fact that normal matter doesn't decay into it, but this argument doesn't rule out the stability of SQM because a similar transition would require a prohibitive number of simultaneous weak decays. Theoretical calculations, chiefly with the MIT bag model, give some support to the idea of SQM stability for a respectable chunk of parameter space, but the question of stability is only answerable experimentally (or observationally), with the experimental signatures being hadronic objects with anomalously low charge to mass ratios or that are considerably more massive than stable nuclei.

Strangelets (small chunks of SQM) may also be metastable and decay with weak lifetimes, but Jack's chief interest was generally in the idea of stable cold SQM. Always part of his talks on the subject was a slide about the exciting implications of stability: the extensions of chemistry and materials with stable nuclei up to charge of $Z \approx 1000$ (the surface electric field strength would prevent higher charge) and superdense materials, the potential as a convenient clean energy source [6]. His interest led him to join Yale colleague Shiva Kumar as part of Experiment 814 at the Brookhaven AGS, where his group performed an initial strangelet search and started their work in heavy-ion physics. He (with Dick Majka as cospokesperson) then led the much more specialized E864, a large-acceptance open-geometry spectrometer with a particularly notable hadronic calorimeter and "Late Energy Trigger" system that facilitated a highly sensitive search [7] that reached a sensitivity of around $10^{-8}$ per 
central AGS heavy-ion collision, for positive, negative and neutral(!) strangelets with $A>5$ and lifetime $\tau>50$ ns.

No strangelets were found by E864, but like many AGS experiments it was great place to be a student-John Lajoie, Zhangbu Xu, Ken Barish and Jamie Nagle (from Shiva's group) are among the Yale $\mathrm{PhD}$ students from E864 who are still very active in the field. In addition, measurements were made of production yields (and limits) for stable and unstable light nuclei, antinuclei and hypernuclei, as summarized in the left panel of Figure 2: and detailed in Refs $[8,9]$. Among other things, these measurements strongly suggested that cold SQM wasn't going to be formed in collisions as the "penalties" for coalescing additional nucleons (a factor of $\approx 48$, as shown by the fit on the positive $A$ side of the Figure) and strangeness (another factor of a couple, though with a large uncertainty) into clusters was too steep. The search for cold SQM would need other avenues...
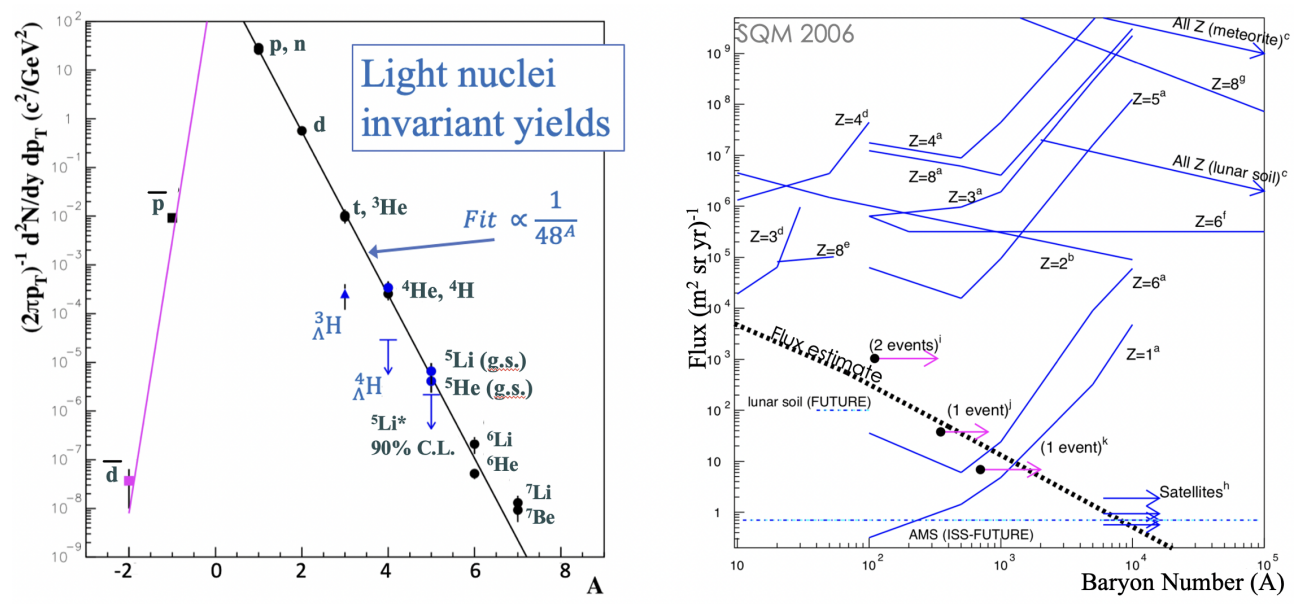

Figure 2. Left panel shows a compilation of some of the banner results from E864: yields and limits for light nuclei, antinuclei, hypernuclei at the AGS, with implications for coalescing strangelets described in text. Right panel gives an overview circa 2006 of searches relevant to strangelets in cosmic rays as strange star relics along with the implied flux. The lunar soil search is long completed [12] and the first published AMS results are recently in [13], to supercede those "future" lines in the figure with sensitivities noted in the text.

After getting reeled in by various interests in heavy-ions at RHIC (see section 3), Jack returned to his interest in strangelets following the 1998 hypothesis of the existence of a "colorflavor-locked" phase of SQM that pointed Jack down the road of astrophysical searches to investigate if "neutron stars" could in fact all be strange stars. (The possibility additional stable SQM phases removed the objection that observed pulsar glitches would not be consistent with fully SQM stars). Under that assumption, theorist Jes Madsen computed a flux estimate [11] for a strangelet component of cosmic rays incident on Earth. Jack then spent some time researching whether that estimated flux could be ruled out by various balloon or satellite-based studies, or relic searches with terrestrial or lunar material (the landscape hasn't changed much since our group looked into it - a still-almost-current plot is in Figure 2 from Ref [10]. As Jack emphasized in his contribution proceedings to the 2003 SQM conference: "...the question as to (stable SQM's) existence remains open. A new experiment, the Alpha Magnetic Spectrometer (AMS), to be mounted on the International Space Station offers the possibility of a definitive answer to the question." The promise of AMS is a tremendously 
high-class detector for cosmic ray measurements sitting largely above the influence of the geomagnetic field. For a strangelet search, it operates in principle much as E864 did, with redundant time-of-flight measurements (to measure speed $\beta$ ) and precise tracking (for a rigidity $R$ ) that could be combined to measure the charge to mass ratio of each cosmic ray. Because the launch of AMS was delayed and for a time uncertain in the aftermath of the Columbia accident, another clever idea of Jack's was to use lunar soil (applied for and granted from among NASA's Apollo 11 samples) as a beam source in Yale's tandem Van-de-graff, which operated as a mass spectrometer for a strangelet search. In particular, this was a follow-up on some interesting events recorded by the AMS-01 prototype flight. The sensitivities achieved by the analysis of Ke Han [12] from Jack's group translate to a flux of around 200 per $\left(\mathrm{m}^{2}\right.$ sr yr) over a somewhat narrow bite in mass of $40<A<70$, and so were sufficient to begin address the flux predictions and the AMS-01 events, but not at the level that AMS would promise. And at last in 2021, the long-awaited first results from AMS on strangelets are published [13]- no observation of strangelets, with a flux sensitivity of around 40 per $\left(\mathrm{m}^{2} \mathrm{sr}\right.$ yr) over a large range of charge and mass. The limit sits below the expected flux in the right panel of Figure 2 for a very significant mass range. With the large uncertainties attached to the expected flux, one shouldn't yet argue that this has succeeded at Jack's expectation being able to rule out the idea of stable SQM, but improvements in the AMS search sensitivity still hold promise to do that (or, indeed, for discovery).

\section{Local Parity Violation}

Leading E864 heightened Jack's interest in the larger heavy-ion physics world, and the arrival at Yale of John Harris drew him the rest of the way in as he joined the STAR experiment. As the RHIC collider was nearing its first run, Jack also had a very lovely idea (as he would point out with a broad smile, that became tenable only after he found a minor error in a paper by some excellent theorists) to measure the charged particle stopping (and through this estimate the energy density achieved) at RHIC via a measurement of Bremstrahlung photons. I really enjoyed this description of the design that I ran across on Ben Monreal's twitter feed soon after Jack's passing: "Start with a cute, elegant bit of classical E\&M phenomenology everyone else overlooked, realize it produces a tiny photon signal that's swamped by pion backgrounds, and concoct a specialized bit of apparatus that enables a signal on/signal off background subtraction. A tiny work of art ". The bremstrahlung experiment didn't go forward at RHIC, bit it has recently seen renewed interest at LHC energy [14]

Though he worked on other topics in heavy-ions, his main interest started with the 1998 paper [15] suggesting that parity and CP symmetries might be broken "locally" in regions generated in heavy-ion collisions. He led the charge to push on what could be done with this idea experimentally, in the early days helping to organize many joint meetings with theorists and experimentalists to try to understand the types of observables that could be formed from combinations of particles' final-state momenta to be sensitive [16], and the methods to extract a reliable measurement given the nature of this "local" violation to reverse handedness from region to region, and later working on complementary methods such as measurements of correlations of $\Lambda$-helicities [17].

While Jack's attention was largely back on strangelets in the mid 2000s, the leap was made forward to understand that this Local Parity Violation should combine with the large magnetic field in heavy-ion events to cause charge separation across the event reaction plane (known as the "Chiral Magnetic Effect") [18] and the methods for observing this were developed [19], and Jack rejoined the effort full-bore as STAR made initial measurements that found signals qualitatively consistent with many aspects of the CME expectations [20]. This was a big step, but as the New York Times noted: "Dr. Sandweiss cautioned that it was 
still possible that some other effect could be mimicking the parity violation". Backgrounds, chiefly from flowing two-particle clusters or similar sources, were known early-on to be a potential issue and have proven very difficult to convincingly separate from CME-related signal despite a great deal of work on the subject.

Perhaps the most promising method to separate signal from background is through the use of isobar collisions that were run by RHIC in 2018. ${ }_{44}^{96} \mathrm{Ru}+{ }_{44}^{96} \mathrm{Ru}$ and ${ }_{40}^{96} \mathrm{Zr}+{ }_{40}^{96} \mathrm{Zr}$ collision systems are expected to have differences in CME-related signal by around $15 \%$ due to the larger magnetic field in $\mathrm{Ru}$, but to have similar backgrounds (with background differences that to first order can be estimated by measuring flow in the two systems). The results from a large-scale effort of several groups within STAR, all performing blind analyses, are due to be released to the public by September 2021, and the field is eagerly awaiting these results. While this work has been going on, there have been some very nice developments in other CME-analysis techniques. One particularly promising method involves utilizing the difference of measured CME signal and flow with respect to the reaction plane in the central region (which will maximize backgrounds) and the event plane measured in forward detectors (which will maximize CME-related signal). Depending on assumptions about how the $\mathrm{CME}$ signal should scale between $\mathrm{Au}+\mathrm{Au}$ to the isobar systems, it can be argued that this method has similar or even higher sensitivity than the isobar method. STAR has recently submitted results from this method for publication [21]. These results are reproduced in Figure ?? and they imply that while the fraction of measured charge separation signal originating from CME is small $(<10 \%)$, it is $1-3$ sigma away from zero, though some concerns about non-flow backgrounds remain. It will be very interesting to see what the isobar results bring to light! Looking to the future, another Jack-inspired idea is to correlate the CME-related charge separation with other parity-odd signals such as net lambda helicity[22]. Such a study holds the promise of eliminating flow-related background but how much the signal will be present in the strange quarks is a significant question.

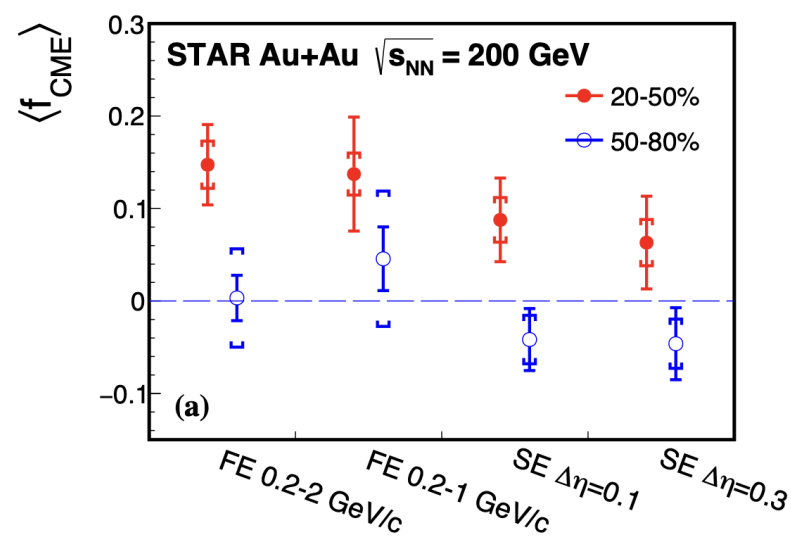

Figure 3. STAR results for the fraction of charge separation due to CME ( $f_{C M E}$ utilizing the difference in various signals with respect to forward and central reaction planes. Different columns represents variations in the methods, using full (FE) or sub-events (SE) for the analysis. The resulting signal is consistent with zero in peripheral collisions but 1-3 sigma away from zero in mid-central collisions. There are many important nuances here-see [21] for details. 


\section{Summary}

The intention of the organizers was that we remember Jack by discussing some of the physics that particularly delighted him, and it is appropriate that we're talking about him as the STAR isobar results near completion and the AMS strangelet limits are released. We keenly miss having him with us to discuss these new results that he would have been eagerly awaiting, but that's truly only a small piece of what we miss about Jack!

\section{References}

[1] D H Stork, R W Birge, R P Haddock, L T Kerth, J Sandweiss and M N Whitehead, Phys Rev 105, 729 (1957)

[2] R W Birge, R P Haddock, L T Kerth, J Sandweiss, D H Stork and M N Whitehead, Il Nuovo Cimento 4, 351 (1956)

[3] J Sandweiss, Physics Today 31, 10 (1978)

[4] A R Bodmer, Phys Rev D 4, 1601 (1971)

[5] E Witten, Phys Rev D 30, 272 (1984)

[6] G L Shaw, M Shin, R Dalitz and M Desai, Nature 337, 436 (1989)

[7] T A Armstrong et. al. (E864 Collaboration), Phys Rev C 63, 054903 (2001)

[8] T A Armstrong et. al. (E864 Collaboration), Phys Rev Lett 83, 5431 (1999)

[9] T A Armstrong et. al. (E864 Collaboration), Phys Rev C 67, 014902 (2004)

[10] J Sandweiss, J. Phys G, 30, S51 (2004), E Finch, J. Phys G, 32, S251 (2006)

[11] J Madsen, Phys Rev D 71, 014026 (2005)

[12] K Han et. al. (LSSS Collaboration), Phys Rev Lett 103, 092302 (2009)

[13] M Aguilar et. al. (AMS Collaboration), Physics Reports 894, 1 (2021)

[14] S Park and U Wiedemann, arXiv:2107.05129-hep-ph (2021)

[15] D Kharzeev, R D Pisarski, M H G Tytgat, Phys Rev Lett 81, 512 (1998)

[16] L E Finch, A Chikanian R S Longacre, J Sandweiss and J H Thomas, Phys Rev C 65, 014908 (2002)

[17] F Du, L E Finch and J Sandweiss, Phys Rev C 78, 044908 (2008)

[18] K Fukushima, D E Kharzeev and H J Warringa, Phys Rev D 78, 074033 (2008)

[19] S Voloshin, Phys Rev C 70, 057901 (2004)

[20] B I Abelev et al (STAR Collaboration), Phys Rev Lett 103, 251601 (2009)

[21] M Abdallah et al (STAR Collaboration), arXiv:2106.09243-nucl-ex (2021)

[22] L E Finch and S J Murray, Phys Rev C 96, 044911 (2017) 